\title{
Extending the Depth of Field in Aberration-Corrected STEM by 3D Sectioning
}

\author{
Robert Hovden, ${ }^{*}$ Huolin L. Xin, ${ }^{* *}$ and David A. Muller* \\ * School of Applied and Engineering Physics, Cornell University, Ithaca, NY 14853 \\ ** Department of Physics, Cornell University, Ithaca, NY 14853
}

Aberration-corrected scanning transmission electron microscopes (STEM) provide subangstrom resolution; however, the large convergence angle greatly reduces the depth of focus. For microscopes with a small depth of focus, information outside of the focal plane quickly becomes blurred and less defined [1]. This effect enables depth-sectioning reconstruction but can be troublesome for some applications as it may not be possible to image a sample entirely in focus. Extended depth-of-field techniques, however, allow a single image with all parts of the image being in-focus to be extracted from a series of images focused at a range of depths (Fig. 1). In recent years a variety of algorithmic approaches have been employed for bright field optical microscopy. Here, we demonstrate that established optical microscopy methods can be utilized to extend the $\sim 6 \mathrm{~nm}$ depth of focus of an aberration-corrected Nion UltraSTEM (33 mrad, 100keV) to image Pt-Co nanoparticles on a thick carbon black support. These techniques allow us to automatically obtain a single image with all the particles in focus as well as a topography map for qualitative analysis.

The nanoparticles are $\sim 4.6 \mathrm{~nm}$ in diameter and spaced well over a $\sim 100 \mathrm{~nm}$ spherical carbonblack support, making it difficult for the microscope, with a depth of field $\sim 5.8 \mathrm{~nm}$, to acquire an image with both the particles on the top and the bottom surfaces in focus (Fig. 2a. and 2b.). We can extend the depth of field by moving the focal plane along the optical axis and acquiring images at each step, obtaining a z-stack [1].The challenge then becomes extracting the in-focus information out of each image in the stack automatically. That is, we would like to combine or fuse the image stack to a single two-dimensional image with an apparent extended depth of field.

Extended depth of field techniques used in light microscopy can be applied to HAADF electron microscopy fairly readily. There are three basic approaches to fusing an image stack and obtaining an extended depth of field: A point process basis, an area process basis, and those that utilize the frequency space of the image [2]. These approaches provide a $z$-height selection rule for each $x-y$ location in the image stack. A classic example of an area approach takes the variance of intensities in the neighborhood. Larger variance of intensities is assumed to be in focus and a $\mathrm{z}$ height can be selected (shown in Fig 2d). It has gained attention because of its computational simplicity and relative effectiveness. The frequency or wavelet approach has claimed success by many groups [2-4]. These techniques analyze the frequency components of the image at various locations by using processes such as a wavelet or windowed Fourier transform. In focus regions are assumed to have sharp details and thus contain high frequency information. The results of a complex wavelet algorithm (see Forster et al. [4]) are shown in Fig 2f. Lastly, in the model-based deconvolution developed by F. Aguet et al. [5] the image stack is assumed to be a convolution of the point spread function with a texture mapped to a thin surface. A least-squares solution produces a topographic and texture map that is used in extending the image depth of field. The resulting image is shown in Fig $2 \mathrm{f}$.

Extended depth-of-field algorithms provide exceptional qualitative results with disperse PtCo nanoparticles as imaged in an aberration-corrected STEM. Additionally, such algorithms can provide accurate topographic maps of particles (Fig. 2c, as generated from wavelet techniques) that allow quantitative analysis of the particle z-height.

[1] Xin, H. L. and Muller, D. A., Journal of Electron Microscopy 58, 157-165, (2009). 
[2] Valdecasas, A. G., D. Marshall, et al, Micron 32(6): 559-569 (2001)

[3] Unser, M. and A. Aldroubi, Proceedings of the IEEE 84(4): 626-638 (1996)

[4] Forster, B., D Van de Ville, et al, Microscopy Research and Technique 65(1-2): 33-42 (2004)

[5] Aguet, F., D. V. De Ville, et al., IEEE Transactions on Image Processing 17(7): 1144 (2008)

[6] Supported by the Semiconductor Research Corporation and NSF MRSEC (DMR 0520404)
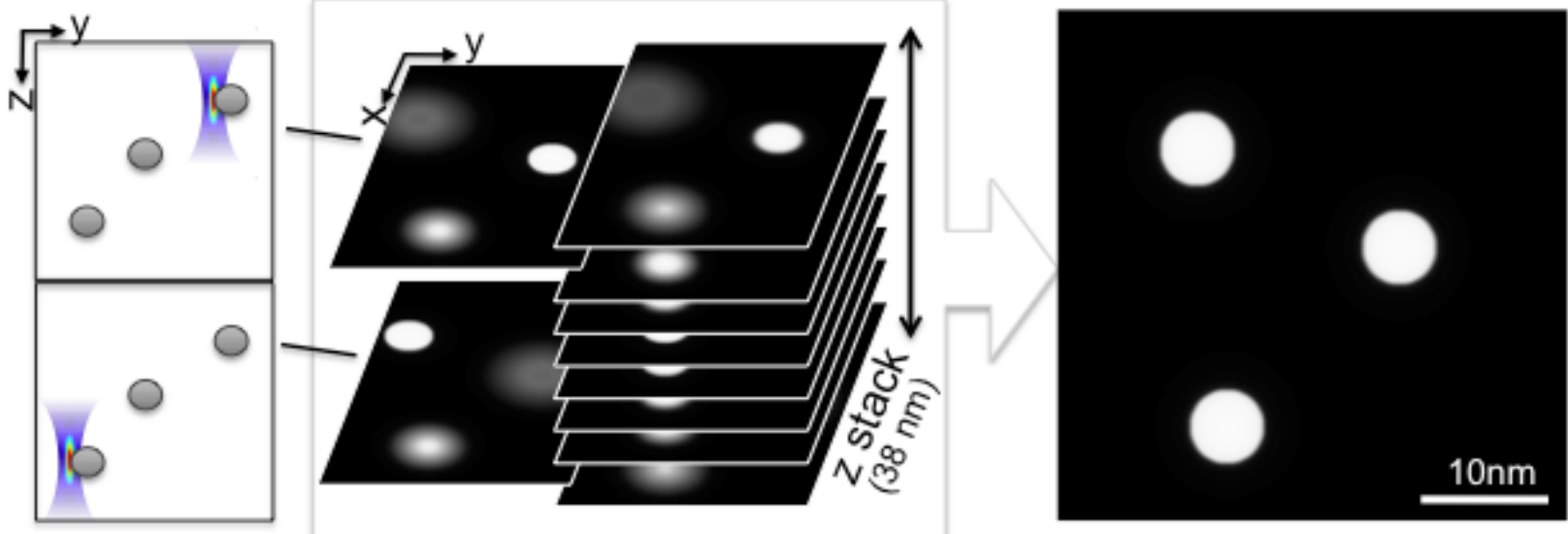

FIG. 1: Simulated particles at different heights $(0,15,30 \mathrm{~nm})$ are imaged over a range of defocuses (-4 to $34 \mathrm{~nm}$ ) by a $100 \mathrm{keV}, 33 \mathrm{mrad}$ probe. Due to the probes limited depth of field (shown left), particles go in and out of focus. By fusing the in-focus information from each image in the stack, we can effectively extend the depth of focus (shown right).
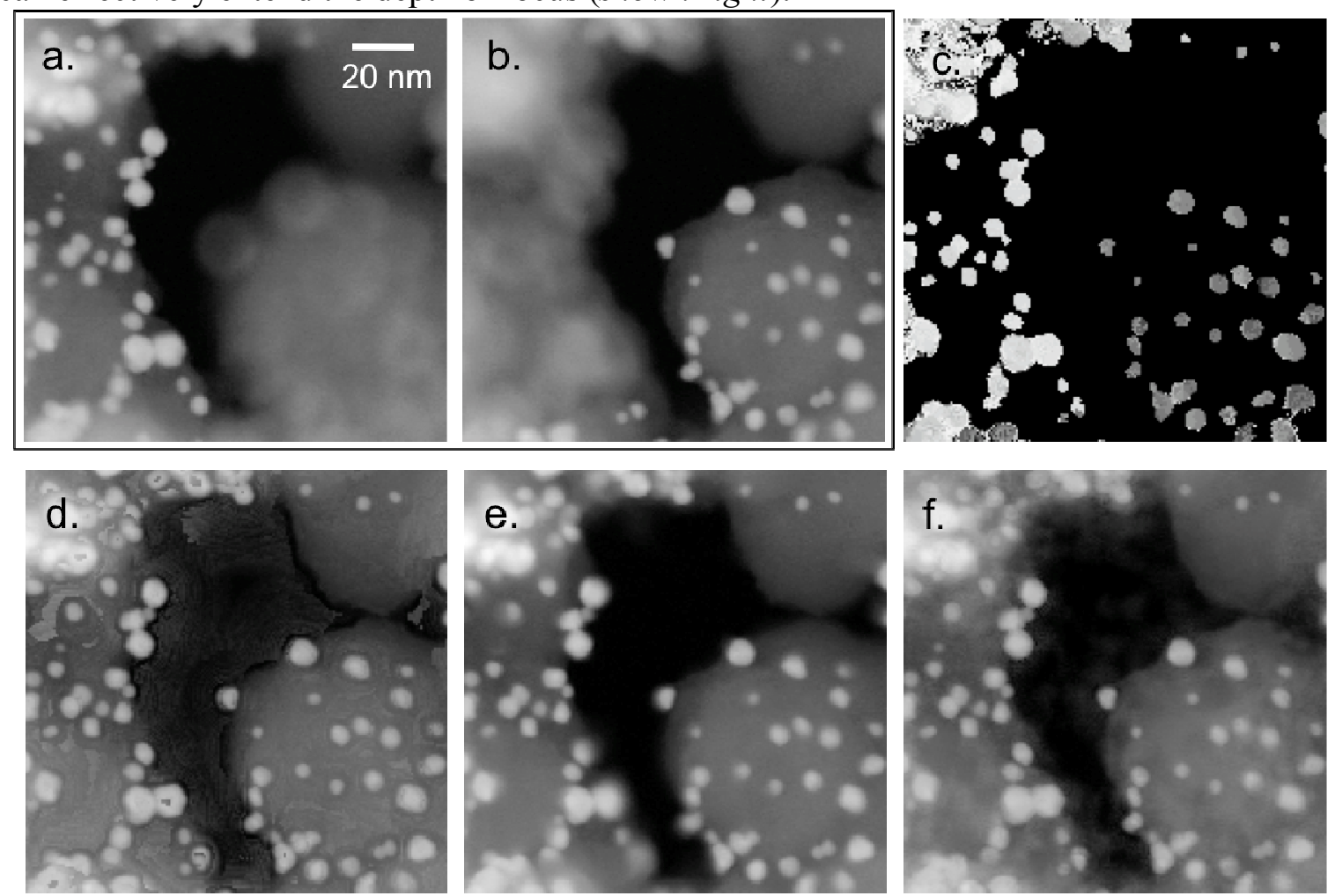

FIG. 2: PtCo nanoparticles imaged by an aberration-corrected Nion (100keV, 33mrad). a) and b) shows two regions with different focal planes with the corresponding particle topography shown in c). The depth of field was extended using: d) variance method, e) model based deconvolution, and f) wavelet transforms. 